\title{
Analysis of the Complex Light Field Generated by a Deflectable Mirror Array Device
}

\author{
Erdem Ulusoy, Levent Onural, Haldun M. Özaktaş \\ Department of Electrical and Electronics Engineering Bilkent University, TR-06800, Ankara, \\ TURKEY
}

\begin{abstract}
An exact analysis of the scalar coherent monochromatic light field produced by a deflectable mirror array device is presented. The three-dimensional light field is related to the tilt angles of the mirrors. The first RayleighSommerfeld diffraction formula is used to model the diffraction. The analysis is carried out based on the assumption that the mirrors can be tilted with continuously varying angles, so the field produced by a finite (discrete) set of possible tilt angles is included as a special case.
\end{abstract}

Keywords: Deflectable mirror array device, Three-dimensional light field synthesis, First Rayleigh-Sommerfeld diffraction formula

\section{INTRODUCTION}

The deflectable mirror array device (DMAD) is a reflection-mode spatial light modulator (SLM), which consists of a two-dimensional array of square shaped identical micro-mirrors, which can be tilted along their diagonal axis through application of electrical signals to appropriate control electrodes. The deflection of each mirror can be controlled separately. [1]

One of the most widely used DMADs is the digital micromirror device (DMD) by Texas Instruments. [2] Although it is originally designed for use in digital video display systems [3], it is occasionally employed in holography applications. In [4], it is used to generate holographic stereograms. In [5], its usage in digital holographic interferometry is proposed.

In this work, we analyze the light field synthesized by a generic DMAD. The light field is related to the tilt angles of the mirrors. We investigate the generation of monochromatic light fields from plane-wave illumination with the same wavelength. The analysis is based on the scalar wave theory of light. To model diffraction, we used the first Rayleigh-Sommerfeld diffraction formula, which does not make any kind of paraxial, Fresnel, or Fraunhofer approximations. In the event that the rotation angles of mirrors are large, due to the significant overlap between the diffraction space of a mirror with the DMAD itself, it would not be possible to add individual isolated contributions from the mirrors to each other to obtain the total field. We assumed that the tilt angles of the mirrors are small enough so the field produced by the individual mirrors can be superposed. Moreover, we assumed that the mirrors are perfect reflectors, while the spacing between them are perfect absorbers. Apart from these assumptions, the analysis is exact. We place no restriction on the tilt angles and assume continuous tuning is possible. Therefore, the analysis is also valid when tuning only among a finite discrete set of angles is possible, as in a DMD.

\section{NOTATION AND CONVENTIONS}

\subsection{Chosen Coordinate System and Illumination Wave}

To express the phasor of the monochromatic scalar light field generated by a DMAD upon illumination with a plane wave as a function of the space coordinates, a fixed and unique coordinate system should be chosen. Our choice of this coordinate system is shown in fig. 1(a). The main motivation underlying this choice is that the mirrors of the DMAD are tilted along their diagonal axes, so the results will be more elegant and manageable with these axes. We assume that in the chosen coordinate system, the tilt axes of mirrors are parallel to the $y$-axis. Tilt angles are measured from the $x$-axis towards the $z$-axis.

This work is supported by EC within FP6 under Grant 511568 with the acronym 3DTV.

Holography 2005: Intl. Conf. on Holography, Optical Recording, and Processing of Information,

Yury Denisyuk, Ventseslav Sainov, Elena Stoykova, Eds., Proceedings of SPIE Vol. 6252,

62521L, (2006) -0277-786X/06/\$15 - doi: 10.1117/12.677163

Proc. of SPIE Vol. $625262521 \mathrm{~L}-1$ 


\subsection{Indexing the mirrors}

The width of the mirrors is taken to be $2 W$ while the spacing between them is $L$. From fig. 1 (b), we see that mirrors are centered on a two-dimensional periodic lattice characterized by the orthogonal vectors $s\left[\begin{array}{ccc}-1 & 1 & 0\end{array}\right]^{T}$ and $s\left[\begin{array}{lll}1 & 1 & 0\end{array}\right]^{T}$ where $s=\left(W \sqrt{2}+\frac{L \sqrt{2}}{2}\right)$. Therefore, mirrors are conveniently indexed with a $2 \times 1$ vector i such that the coordinates of the center of the mirror indexed by $\mathbf{i}=\left[\begin{array}{ll}m & n\end{array}\right]^{T}$ are given by:

$$
\mathbf{T}_{\mathbf{i}}=\left[\begin{array}{c}
x_{c} \\
y_{c} \\
0
\end{array}\right]=\left(W \sqrt{2}+\frac{L \sqrt{2}}{2}\right)\left[\begin{array}{cc}
-1 & 1 \\
1 & 1 \\
0 & 0
\end{array}\right] \mathbf{i}
$$

\subsection{Functional Representation of the Illuminating Wave and Generated Fields}

The illumination of thewhole DMAD is accomplished with a single monochromatic plane wave of wavelength $\lambda$, whose functional representation in the coordinate system of fig. 1(a) is $\exp \left\{j \frac{2 \pi}{\lambda} \kappa^{T} \mathbf{x}\right\}$ where $\mathbf{x}$ is the position vector given by $\mathbf{x}=\left[\begin{array}{lll}x & y & z\end{array}\right]^{T}$ and $\kappa$ is the direction cosines vector $\kappa=\left[\begin{array}{ccc}\alpha & \beta & \gamma\end{array}\right]^{T}$ with $\alpha^{2}+\beta^{2}+\gamma^{2}=1$. [1]

Upon illumination with this wave, each mirror will generate a reflected field. We call the function that represents the light field (in the coordinate system of fig. $1(a)$ ) generated by the $i$ 'th mirror, which is rotated by an angle $\theta_{\mathbf{i}}$ as $\phi_{\kappa}^{\left(\mathbf{i}, \theta_{\mathbf{i}}\right)}(\mathbf{x})$.

Therefore, the total field produced by the DMAD will be given by $u(\mathbf{x})=\sum_{\mathbf{i}} \phi_{\kappa}^{\left(\mathbf{i}, \theta_{\mathbf{i}}\right)}(\mathbf{x})$

\section{ANALYSIS}

The main purpose of this section is to find, in the coordinate system of fig. 1(a), the phasor of the monochromatic field generated by an arbitrary mirror of the DMAD indexed by $\mathbf{i}$, when it is tilted by an angle $\theta_{\mathbf{i}}$, which we previously denoted as $\phi_{\kappa}^{\left(\mathbf{i}, \theta_{\mathbf{i}}\right)}(\mathbf{x})$. Towards this end, we will consider the four steps below.

\subsection{Field Generated by a Single Mirror whose Edges are Parallel to the Coordinate Axes}

We first consider the solution of the simple problem shown in fig. 1(c). We assume that the mirror is illuminated by a plane wave $\exp \left\{j \frac{2 \pi}{\lambda} \kappa^{T} \mathbf{x}\right\}$. We denote the functional form of the reflected and diffracted light (solution of the problem posed in fig. $1(\mathrm{c}))$ by

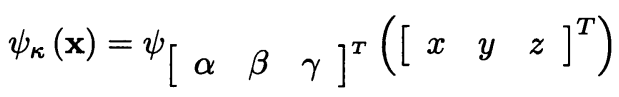

which will be essential in the analysis. As seen, this is a 6-parameter function.

While expressing the field generated by any arbitrary mirror of the DMAD in the coordinate system of fig. 1(a), we will employ this function by properly modifying its parameters $\kappa$ and $\mathbf{x}$. In particular, we will express $\phi_{\kappa}^{\left(\mathbf{i}, \boldsymbol{\theta}_{\mathbf{i}}\right)}(\mathbf{x})$ in terms of this function $\psi$.

To obtain explicit expressions for the $\psi$ function, first note that the field across the mirror's aperture is given by:

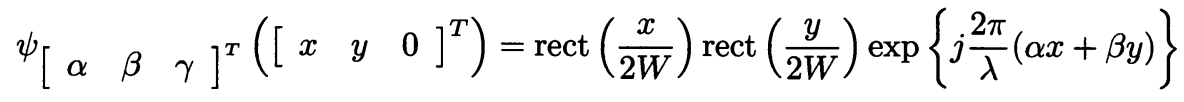

Using the first Rayleigh-Sommerfeld diffraction formula [1], we obtain

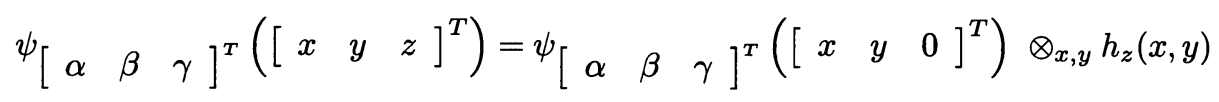


where the Rayleigh-Sommerfeld diffraction kernel is given by [1]:

$$
h_{z}(x, y)=\frac{z}{j \lambda} \frac{\exp \left\{j \frac{2 \pi}{\lambda} \sqrt{x^{2}+y^{2}+z^{2}}\right\}}{x^{2}+y^{2}+z^{2}}
$$

Here, $\otimes_{x, y}$ denotes two-dimensional convolution operation.

In the spatial frequency domain, we have the following relation [1]:

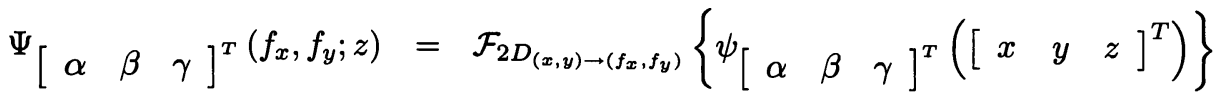

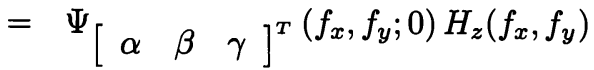

where $H_{z}\left(f_{x}, f_{y}\right)$ is the two-dimensional Fourier transform of $h_{z}(x, y)$ given by [1]:

$$
H_{z}(x, y)=\exp \left\{j 2 \pi \frac{z}{\lambda} \sqrt{1-\left(\lambda f_{x}\right)^{2}-\left(\lambda f_{y}\right)^{2}}\right\} \operatorname{circ}\left(\sqrt{\left(\lambda f_{x}\right)^{2}+\left(\lambda f_{y}\right)^{2}}\right)
$$

Upon taking the two-dimensional Fourier transform of Eq. 3 and multiplying it with Eq. 7, we get

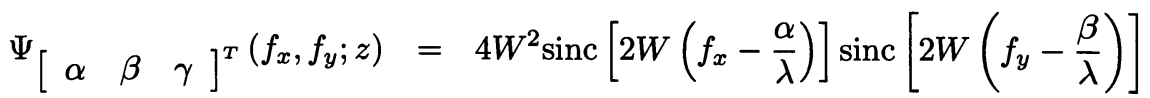

$$
\begin{aligned}
& \exp \left\{j 2 \pi \frac{z}{\lambda} \sqrt{1-\left(\lambda f_{x}\right)^{2}-\left(\lambda f_{y}\right)^{2}}\right\} \operatorname{circ}\left(\sqrt{\left(\lambda f_{x}\right)^{2}+\left(\lambda f_{y}\right)^{2}}\right)
\end{aligned}
$$

\subsection{Untilted Mirror at Origin}

Secondly, we will express $\phi_{\kappa}^{(\mathbf{0}, 0)}(\mathbf{x})$ in terms of the $\psi$ function of Eq. 2. The problem is depicted in fig. 1(d). Here, $(x, y, z)$ is the coordinate system of fig. $1(\mathrm{a})$; and $\left(x^{\prime}, y^{\prime}, z^{\prime}\right)$ is a useful intermediate coordinate system. Position vectors are given as $\mathbf{x}$ and $\mathbf{x}^{\prime}$ and the unique illumination plane wave has the functional forms $\exp \left\{j \frac{2 \pi}{\lambda} \kappa^{T} \mathbf{x}\right\}$ and $\exp \left\{j \frac{2 \pi}{\lambda} \kappa^{\prime T} \mathbf{x}^{\prime}\right\}$ with respect to these two coordinate systems respectively. The following relations exist between the coordinates and the parameters:

$$
\begin{aligned}
& \mathbf{x}^{\prime}=\mathbf{R}_{0} \mathbf{x} \\
& \kappa^{\prime}=\mathbf{R}_{0} \kappa
\end{aligned}
$$

where

$$
\mathbf{R}_{0}=\left[\begin{array}{ccc}
\frac{1}{\sqrt{2}} & \frac{1}{\sqrt{2}} & 0 \\
-\frac{1}{\sqrt{2}} & \frac{1}{\sqrt{2}} & 0 \\
0 & 0 & 1
\end{array}\right]
$$

The reason for denoting the rotation matrix of Eq. 11 by $\mathbf{R}_{0}$ will become clear in the next subsection.

From the previous subsection and Eq. 2, it can be recognized that the functional expression of the synthesized light field, in $\left(x^{\prime}, y^{\prime}, z^{\prime}\right)$ coordinates, is given by

$$
\psi_{\kappa^{\prime}}\left(\mathbf{x}^{\prime}\right)
$$

By applying the transformations of Eq. 9 and Eq. 10 to Eq. 12 we can find the functional form of the created field in the $(x, y, z)$ coordinates, as

$$
\phi_{\kappa}^{(\mathbf{0}, 0)}(\mathbf{x})=\psi_{\mathbf{R}_{0} \kappa}\left(\mathbf{R}_{0} \mathbf{x}\right)
$$




\subsection{Tilted Mirror at Origin}

Thirdly, we will express $\phi_{\kappa}^{\left(\mathbf{0}, \theta_{\mathbf{0}}\right)}(\mathbf{x})$ in terms of the $\psi$ function. This problem is shown in fig. 1(e). Here, $(x, y, z)$ is the coordinate system of fig. 1(a); and $\left(x^{\prime}, y^{\prime}, z^{\prime}\right)$ is another useful intermediate coordinate system. Respectively, the position vectors are given as $\mathbf{x}$ and $\mathbf{x}^{\prime}$ and the unique illumination plane wave has the functional forms $\exp \left\{j \frac{2 \pi}{\lambda} \kappa^{T} \mathbf{x}\right\}$ and $\exp \left\{j \frac{2 \pi}{\lambda} \kappa^{\prime T} \mathbf{x}^{\prime}\right\}$ with respect to these coordinate systems. The following relations exist between the coordinates and the parameters:

$$
\begin{aligned}
\mathbf{x}^{\prime} & =\mathbf{K}_{\theta_{\mathbf{0}}} \mathbf{x} \\
\kappa^{\prime} & =\mathbf{K}_{\theta_{\mathbf{0}}} \kappa
\end{aligned}
$$

where

$$
\mathbf{K}_{\theta}=\left[\begin{array}{ccc}
\cos \theta & 0 & \sin \theta \\
0 & 1 & 0 \\
-\sin \theta & 0 & \cos \theta
\end{array}\right]
$$

From the previous subsection and Eq. 13, in $\left(x^{\prime}, y^{\prime}, z^{\prime}\right)$ coordinates, we observe that the generated field has the functional form

$$
\phi_{\kappa^{\prime}}^{(\mathbf{0}, 0)}\left(\mathbf{x}^{\prime}\right)=\psi_{\mathbf{R}_{0} \kappa^{\prime}}\left(\mathbf{R}_{0} \mathbf{x}^{\prime}\right)
$$

Applying the transformations in Eq. 14 and Eq. 15 to Eq. 17, we recognize the functional form of the created field in $(x, y, z)$ coordinates as

$$
\begin{aligned}
\phi_{\kappa}^{\left(\mathbf{0}, \theta_{\mathbf{0}}\right)}(\mathbf{x}) & =\phi_{\mathbf{K}_{\theta_{0} \kappa} \kappa}^{(\mathbf{0}, 0)}\left(\mathbf{K}_{\theta_{0}} \mathbf{x}\right) \\
& =\psi_{\mathbf{R}_{0} \mathbf{K}_{\theta_{0}} \kappa}\left(\mathbf{R}_{0} \mathbf{K}_{\theta_{\mathbf{0}}} \mathbf{x}\right) \\
& =\psi_{\mathbf{R}_{\theta_{0}} \kappa}\left(\mathbf{R}_{\theta_{0}} \mathbf{x}\right)
\end{aligned}
$$

where, for notational convenience, multiplication of the matrices of Eq. 11 and Eq. 16 is given a combined notation $\mathbf{R}_{\theta}$, such that

$$
\mathbf{R}_{\theta}=\mathbf{R}_{0} \mathbf{K}_{\theta}=\left[\begin{array}{ccc}
\frac{1}{\sqrt{2}} \cos \theta & \frac{1}{\sqrt{2}} & \frac{1}{\sqrt{2}} \sin \theta \\
-\frac{1}{\sqrt{2}} \cos \theta & \frac{1}{\sqrt{2}} & -\frac{1}{\sqrt{2}} \sin \theta \\
-\sin \theta & 0 & \cos \theta
\end{array}\right]
$$

\subsection{Tilted Mirror Translated From Origin}

Finally, we will express $\phi_{\kappa}^{\left(\mathbf{i}, \theta_{\mathbf{i}}\right)}(\mathbf{x})$ in terms of the $\psi$ function. This problem is shown in fig. 1(f). As before, $(x, y, z)$ is the coordinate system of fig. $1(\mathrm{a}) .\left(x^{\prime}, y^{\prime}, z^{\prime}\right)$ coordinate system corresponds to translation of $(x, y, z)$ coordinate system to the central location of mirror i. Then, through Eq. 1, the position vectors $\mathbf{x}$ and $\mathbf{x}^{\prime}$ are related by:

$$
\mathbf{x}=\mathbf{T}_{\mathbf{i}}+\mathbf{x}^{\prime}
$$

Let us assume that the unique illumination plane wave is represented by functions $A \exp \left\{j \frac{2 \pi}{\lambda} \kappa^{T} \mathbf{x}\right\}$ and $A^{\prime} \exp \left\{j \frac{2 \pi}{\lambda} \kappa^{\prime T} \mathbf{x}^{\prime}\right\}$ in the two coordinate systems respectively. As there is only translation between the two coordinate systems, direction cosines do not change, yielding $\kappa=\kappa^{\prime}$. However, amplitudes should differ by a constant phase term to compensate for the translation. This relates the complex amplitudes of the phasors in the two coordinate systems as $A^{\prime}=A \exp \left\{j \frac{2 \pi}{\lambda} \kappa^{T} \mathbf{T}_{\mathbf{i}}\right\}$. Previously, we have assumed unit amplitude plane waves, hence we take $A=1$ again. Hence, the expression for the illuminating plane wave in $(x, y, z)$ coordinates becomes $\exp \left\{j \frac{2 \pi}{\lambda} \kappa^{T} \mathbf{x}\right\}$, while in $\left(x^{\prime}, y^{\prime}, z^{\prime}\right)$, this expression turns out to be $\exp \left\{j \frac{2 \pi}{\lambda} \kappa^{T} \mathbf{T}_{\mathbf{i}}\right\} \exp \left\{j \frac{2 \pi}{\lambda} \kappa^{T} \mathbf{x}^{\prime}\right\}$.

Exploiting the results of the previous subsection once more, and referring this time to Eq. 18, we see from the equivalence of the problems and coordinate systems that the reflected field, in $\left(x^{\prime}, y^{\prime}, z^{\prime}\right)$ coordinates, is given by

$$
\exp \left\{j \frac{2 \pi}{\lambda} \kappa^{T} \mathbf{T}_{\mathbf{i}}\right\} \phi_{\kappa}^{\left(\mathbf{0}, \theta_{\mathbf{i}}\right)}\left(\mathbf{x}^{\prime}\right)
$$


Inserting Eq. 20 into Eq. 18 and Eq. 21, we recognize that

$$
\begin{aligned}
\phi_{\kappa}^{\left(\mathbf{i}, \theta_{\mathbf{i}}\right)}(\mathbf{x}) & =\exp \left\{j \frac{2 \pi}{\lambda} \kappa^{T} \mathbf{T}_{\mathbf{i}}\right\} \phi_{\kappa}^{\left(\mathbf{0}, \theta_{\mathbf{i}}\right)}\left(\mathbf{x}-\mathbf{T}_{\mathbf{i}}\right) \\
& =\exp \left\{j \frac{2 \pi}{\lambda} \kappa^{T} \mathbf{T}_{\mathbf{i}}\right\} \psi_{\mathbf{R}_{\theta_{\mathbf{i}}} \kappa}\left(\mathbf{R}_{\theta_{\mathbf{i}}}\left(\mathbf{x}-\mathbf{T}_{\mathbf{i}}\right)\right)
\end{aligned}
$$

which is the most general case we set out to handle.

\section{TOTAL COMPLEX LIGHT FIELD PRODUCED BY DMAD}

By virtue of Eq. 22 and the superposition assumption of section Eq. 1, the light field produced by the DMAD, upon incident illumination by the monochromatic plane wave $\exp \left\{j \frac{2 \pi}{\lambda} \kappa^{T} \mathbf{x}\right\}$ is:

$$
u(\mathbf{x})=\sum_{\mathbf{i}} \phi_{\kappa}^{\left(\mathbf{i}, \theta_{\mathbf{1}}\right)}(\mathbf{x})=\sum_{\mathbf{i}} \exp \left\{j \frac{2 \pi}{\lambda} \kappa^{T} \mathbf{T}_{\mathbf{i}}\right\} \psi_{\mathbf{R}_{\theta_{\mathbf{1}}} \kappa}\left(\mathbf{R}_{\theta_{\mathbf{1}}}\left(\mathbf{x}-\mathbf{T}_{\mathbf{i}}\right)\right)
$$

This result is for the coordinate system depicted in fig. 1(a). Explicit expressions of matrices $\mathbf{T}_{\mathbf{i}}$ and $\mathbf{R}_{\theta}$ are provided in Eq. 1 and Eq. 19, respectively. Explicit expressions for the 6-parameter function $\psi_{\kappa}(\mathbf{x})$ of Eq. 2 are discussed in subsection Eq. 3.1.

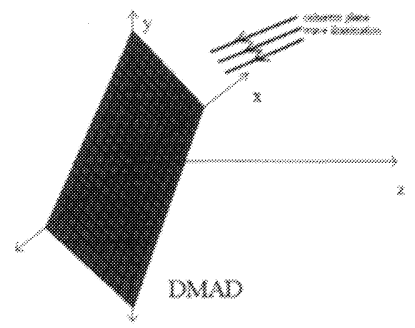

(a)

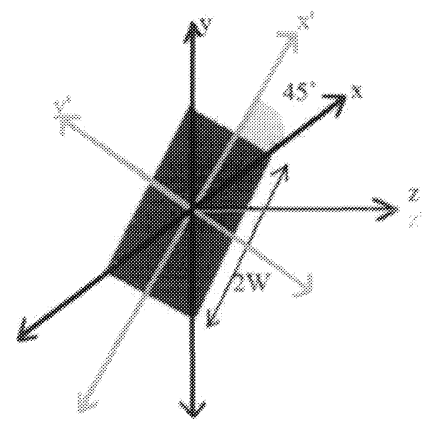

(d)

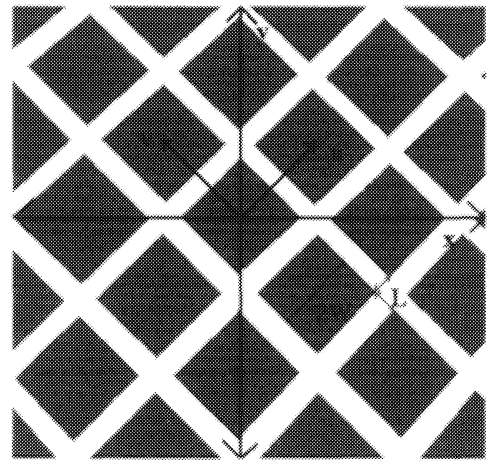

(b)

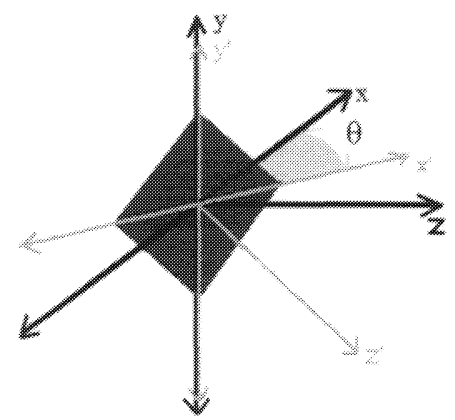

(e)

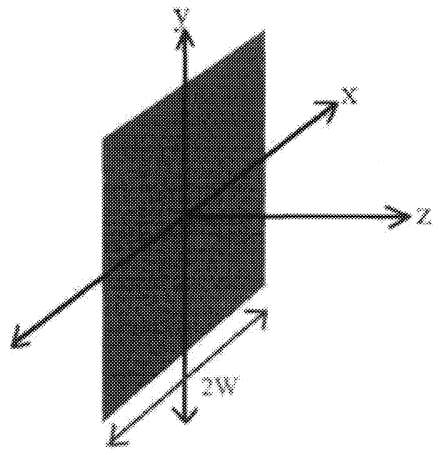

(c)

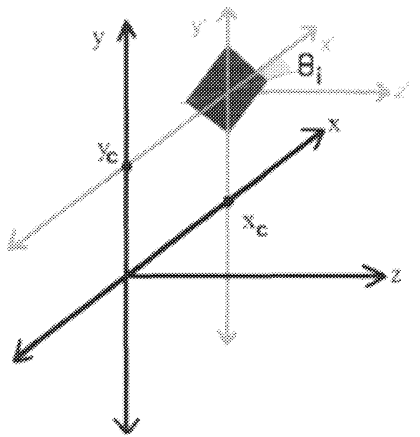

(f)

Figure 1. (a) Coordinate system selected for the analysis of the light field by DMAD (b) Top view of DMAD in the coordinate system of fig. 1(a) (c) Problem analyzed in sec. 3.1. Mirror edges are parallel to coordinate axes. (d) Problem analyzed in sec. 3.2. (e) Problem analyzed in sec. 3.3. (f) Problem analyzed in sec. 3.4. 


\section{REFERENCES}

1. J. W. Goodman. Introduction to Fourier Optics. Mc-Graw-Hill, New York, 1996.

2. R. J. Gove. DMD display systems: the impact of an all digital display. In Int Symp Society for Information Display, page http://www.ti.com/dlp/docs/developer/resources/white/index.shtml. Texas Instruments White Pages, 1994.

3. L. J. Hornbeck. Digital light processing update: status and future applications. In Conf on Projection Displays V, Proc SPIE, volume 3634, pages 158-170, 1999.

4. R. S. Nesbitt, S. L. Smith, R. A. Molnar, and S. A. Benton. Holographic recording using a digital micromirror device. In S. A. Benton, editor, Conf on Practical Holography XIII, Proc SPIE, volume 3637, pages 12-20, 1999.

5. T. Kreis, P. Aswendt, and R. Hofling. Hologram reconstruction using a digital micromirror device. Optical Engineering, 40:926-933, 2001. 